\section{Overview of glycemic control among admitted patients with diabetes in Tripoli University Hospital}

\author{
Haifa Elhadi Alshwikh ${ }^{1 *}$ and Faiza Hander ${ }^{2}$ \\ ${ }^{1}$ Arab Board for Health Specialization, Libyan Board for Medical Specialties, Tripoli University, \\ Tripoli, Libya \\ ${ }^{2}$ Head of Endocrinology Department, Tripoli University Hospital, Tripoli, Libya
}

\section{Abstract}

Aim: To examine the relationship between the levels of $\mathrm{HbA} 1 \mathrm{c}$ and hospital admission rates.

Methods: We recorded $\mathrm{HbA} 1 \mathrm{c}$ levels of all diabetic patients in Tripoli University Hospital over one year.

Results: The mean $\mathrm{HbA} 1 \mathrm{c}$ was $8.03 \%$, with no difference between males and females. Over half of patients (56.5\%) were admitted through their diabetes was well-controlled. Over half of the patients with type 1 diabetes $(57 / 102,55.9 \%)$ had a high $\mathrm{HbA} 1 \mathrm{c}$ at admission compared to $42.1 \%$ of patients with type 2 , who were mainly admitted with $\mathrm{HbA} 1 \mathrm{c}$ level within the acceptable range set for this study. The HbA1c level was positively and significantly correlated with the length of hospital stay $(R=0.93, p=0.000)$, and was significantly associated with hyperglycemia, diabetic ketoacidosis, coronary artery disease, limb ischemia, cataract, osteomyelitis, and non-alcoholic steatohepatitis.

Conclusion: $\mathrm{HbA} 1 \mathrm{c}$ is correlated significantly with hospitalization in type 1 diabetes but not in type 2.

\section{Introduction}

People with diabetes are admitted to hospitals more frequently than the general population due to poor glycemic control and diabetic complications. They also have longer hospital stays and higher mortality rates. It has been estimated that about one-third of people with diabetes need hospitalization two or more times [1,2].

In the acute setting, assessing glycemic control by testing serum glucose might be unreliable because of stress hyperglycemia. On the other hand, glycated hemoglobin (HbA1c) is an indicator of the average blood glucose concentrations during the preceding two to three months, and so it is a more reliable indicator of the glycemic control status and a predictor of diabetic complications and hospitalization rates [2,3]. It is a convenient test and a well-known biomarker used in clinical practice [4].

It is of interest to study the relationship between HbA1c levels of patients with type 1 or type 2 diabetes and

\begin{abstract}
More Information
*Address for Correspondence: Dr. Haifa Elhadi Alshwikh, Internal medicine specialist, Arab board for health Specialization, Libyan Board for Medical Specialties, Tripoli University, Tripoli, Libya, Email: h.alshwikh@uot.edu.ly; haifaalshwikh@gmail.com

Submitted: May 21, 2021

Approved: February 16, 2022

Published: February 18, 2022
\end{abstract}

How to cite this article: Alshwikh HE, Hander F Overview of glycemic control among admitted patients with diabetes in Tripoli University Hospital. J Cardiol Cardiovasc Med. 2022; 7: 013-016.

DOI: 10.29328/journal.jccm.1001125

Copyright License: @ 2022 Alshwikh HE, et al. This is an open access article distributed under the Creative Commons Attribution License, which permits unrestricted use, distribution, and reproduction in any medium, provided the original work is properly cited.

Check for updates

OPEN ACCESS hospitalization burden, and whether certain complications are associated directly with the levels of HbA1c. In this study, we studied the relationship between hospitalization duration and HbA1c levels in diabetic patients hospitalized for different reasons.

\section{Aims and objectives}

Our aim was to examine the relationship between HbA1c levels and admission rates and identify the specific complications linked directly to poor glycemic control.

\section{Methods and design}

The data were collected retrospectively from the hospitalization records of the medical and surgical departments of Tripoli University Hospital. The sample size was 1037 patients.

The inclusion criteria were age $>16$ years, having diabetes, and with an HbA1c measured on or within three months before admission. Patients were included regardless of the 
cause of hospitalization. As we knew from experience that our hospitalized patients are usually elderly and have multiple comorbidities, an HbA1c level $>7.5 \%$ was considered to represent uncontrolled diabetes, as recommended by the American Diabetes Association.

\section{Statistical analysis}

Descriptive statistics (means and standard deviations, and percent frequencies) were calculated using the Statistical Package for the Social Sciences (SPSS version 21). Frequencies were compared statistically by using the Chi-square test.

Ethics approval: The study protocol was approved by the Administration of Tripoli University Hospital.

\section{Results}

The 1037 diabetic patients included in the study had a mean age of 60.7 years \pm 15.14 standard deviation (range 16-96 years). The male to female ratio was 1.01. Most of the patients (90.2\%) had diabetes type-2, and over half of them (56.5\%) had diabetes for longer than 10 years (Table 1).

According to the HbA1c criterion we used to differentiate between good and poor glycemic control, diabetes was poorly controlled in less than half of the patients $(451,43.5 \%)$, but the frequencies in males and females were practically identical (43.3\% and $43.7 \%$, respectively).

Notably, HbA1c was positively and significantly correlated with the duration of hospital stay (Figure 1).

There were 44 different causes of admission to the hospital. For each cause, we analyzed the frequency of patients with HbA1c $\leq 7.0 \%$ or $>7.0 \%$. Statistical analysis showed that poor glycemic control was positively and significantly associated with the following admission causes: hyperglycemia, diabetic ketoacidosis, coronary heart disease, limb ischemia, and cataract (Table 2). None of the other causes of admission was significantly associated with glycemic control (Supplementary Table 1).

\begin{tabular}{|c|c|c|c|}
\hline \multirow{2}{*}{ Age (years) } & Mean \pm SD & \multicolumn{2}{|c|}{$60.7 \pm 15.14$} \\
\hline & Median (range) & \multicolumn{2}{|c|}{$62.0(16-96)$} \\
\hline \multirow{2}{*}{ Hospital stay duration (days) } & Mean \pm SD & \multicolumn{2}{|c|}{$6.1 \pm 4.58$} \\
\hline & Median (range) & \multicolumn{2}{|c|}{$4.0(1-51)$} \\
\hline \multirow{2}{*}{$\mathrm{HbA} 1 \mathrm{c}(\%)$} & Mean \pm SD & \multicolumn{2}{|c|}{$8.0 \pm 1.25$} \\
\hline & Median (range) & \multicolumn{2}{|c|}{$7.4(4.4-16.2)$} \\
\hline & & $\mathrm{n}$ & $\%$ \\
\hline \multirow{2}{*}{ Sex } & Male & 522 & 50.3 \\
\hline & Female & 515 & 49.7 \\
\hline \multirow{2}{*}{ Diabetes type } & DM 1 & 102 & 9.8 \\
\hline & DM 2 & 935 & 90.2 \\
\hline \multirow{5}{*}{ Duration of diabetes (years) } & Newly diagnosed & 27 & 2.6 \\
\hline & $<1$ & 16 & 1.5 \\
\hline & $1-5$ & 125 & 12.1 \\
\hline & $6-10$ & 283 & 27.3 \\
\hline & $>10$ & 586 & 56.5 \\
\hline
\end{tabular}

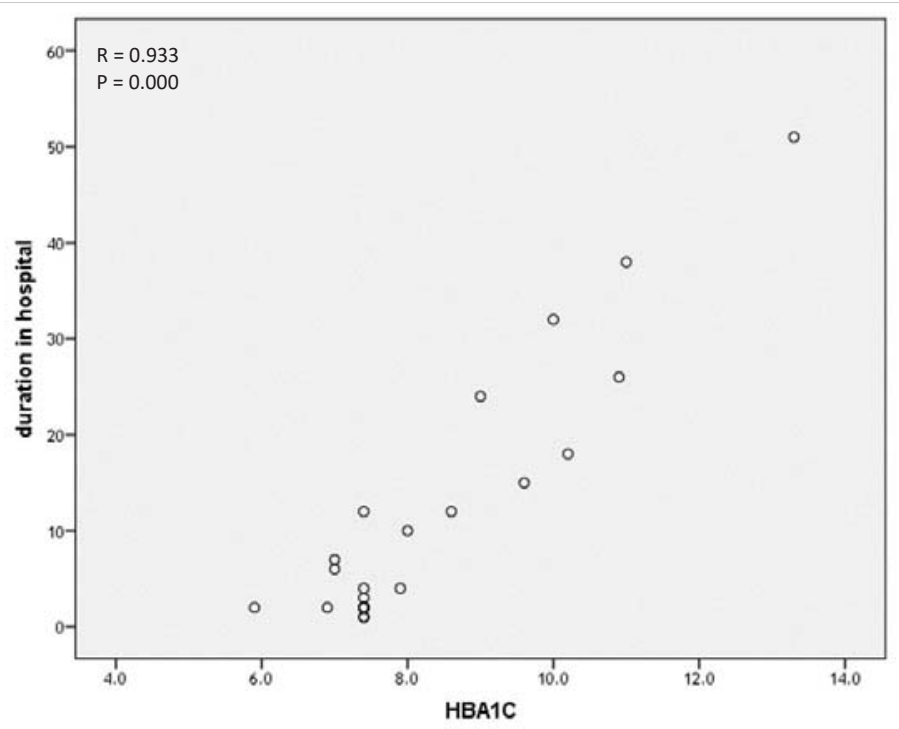

Figure 1: Correlation of $\mathrm{HbA} 1 \mathrm{c}$ level with a duration of hospitalization.

Table 2: Causes of hospital admission found to be positively and significantly associated with the level of glycemic control.

\begin{tabular}{|c|c|c|c|c|c|c|c|c|c|}
\hline \multirow{2}{*}{\multicolumn{2}{|c|}{ Hospitalization cause }} & \multicolumn{2}{|c|}{$\begin{array}{c}\text { HbA1c } \leq \\
7.5 \%\end{array}$} & \multicolumn{2}{|c|}{$\begin{array}{c}\mathrm{HbA} 1 \mathrm{c}> \\
7.5 \%\end{array}$} & \multicolumn{2}{|c|}{ Total } & \multirow[t]{2}{*}{$\mathrm{X}^{2}$} & \multirow[t]{2}{*}{$p$} \\
\hline & & $\mathrm{n}$ & $\%$ & $n$ & $\%$ & $n$ & $\%$ & & \\
\hline \multirow{2}{*}{ Hyperglycemia } & No & 575 & 98.1 & 422 & 93.6 & 997 & 96.1 & \multirow{2}{*}{14.24} & \multirow{2}{*}{0.00} \\
\hline & Yes & 11 & 1.9 & 29 & 6.4 & 40 & 3.9 & & \\
\hline \multirow{2}{*}{$\begin{array}{c}\text { Diabetic } \\
\text { ketoacidosis }\end{array}$} & No & 563 & 96.1 & 415 & 92.0 & 978 & 94.3 & \multirow{2}{*}{7.81} & \multirow{2}{*}{0.005} \\
\hline & Yes & 23 & 3.9 & 36 & 8.0 & 59 & 5.7 & & \\
\hline \multirow{2}{*}{$\begin{array}{c}\text { Coronary artery } \\
\text { disease }\end{array}$} & No & 531 & 90.7 & 384 & 85.2 & 915 & 88.2 & \multirow{2}{*}{9.74} & \multirow{2}{*}{0.002} \\
\hline & Yes & 55 & 9.3 & 67 & 14.8 & 122 & 11.8 & & \\
\hline \multirow{2}{*}{ Limb ischemia } & No & 561 & 95.7 & 417 & 92.5 & 978 & 94.3 & \multirow{2}{*}{5.08} & \multirow{2}{*}{0.024} \\
\hline & Yes & 25 & 4.3 & 34 & 7.5 & 59 & 5.7 & & \\
\hline Cataract & No & 569 & 97.1 & 432 & 95.8 & 1001 & 96.5 & 4.18 & 0.043 \\
\hline
\end{tabular}

\section{Discussion}

The mean $\mathrm{HbA1c}$ level was $8.03 \%$ (range 4.4-16.2\%), and there was no significant difference between males and females. Slightly over half of patients (56.5\%) had good glycemic control (HbA1c $\leq 7.0 \%)$. However, most patients with type-1 diabetes had a high HbA1c level (57/102, $55.9 \%$ ), in contrast to patients with type-2 diabetes, of whom only $42.1 \%$ were admitted with a high $\mathrm{HbA1c}$ level. This indicates that a high HbA1c level could be a better predictor of admission for patients with type 1 diabetes than for type 2 diabetes patients. We believe that these results are quite reliable as most of the patients had type-2 diabetes (90\%) and $56.5 \%$ of them had diabetes for more than 10 years, which makes macrovascular complications the main cause of their admission regardless of their HbA1c levels. This is also supported by previous randomized controlled trials demonstrating that maintaining tight glycemic control does not reduce macrovascular complications in elderly patients with longer duration of diabetes [5-8]. That is also supported by other studies reporting that hospitalization rates were not affected by attaining good glycemic control [9]. In that study, the highest frequency of hospitalization was due to infection in patients with either good (HbA1c 6\% - 7\%) or poor glycemic control (HbA1c 11\%). 
Many previous studies demonstrated that hospitalization rates and HbA1c levels follow a U-shaped or J-shaped relationship, with HgbA1c levels above a certain minimum or below a certain maximum being associated with increased hospitalization risk, whereas maintaining modest glycemic control reduces hospitalization rates. Other studies reported a correlation between high levels of HbA1c and adverse outcomes and stated that poor glycemic control in patients with long-term diabetes increases the risk of hospitalization and mortality. A twofold increased risk of mortality in patients with high levels of HbA1c has been reported [10,11].

A study from Cambridge University Hospitals NHS Foundation Trust showed a non-linear relationship of HbA1c and diabetes with vascular-related admissions: with every $1 \%$ increment in HbA1c level above $7.7 \%$, there was a $6.3 \%$ increased risk of all-cause hospital admission [12].

We found a significant, positive, linear correlation between $\mathrm{HbA1} \mathrm{c}$ and the length of hospital stay. Our finding is supported by studies reporting that hospital stays of six days or more are recorded more frequently for patients with an $\mathrm{HbA1c}$ level of $7 \%$ or more [1].

Our study also investigated the relationship between the causes of admission and the level of HbA1c. Strong correlations were found for certain causes, such as metabolic disorders, including hyperglycemia and diabetic ketoacidosis. High levels of HbA1c and poor glycemic control were also associated with coronary artery disease, limb ischemia, cataract, osteomyelitis, and non-alcoholic steatohepatitis. The association between cardiovascular risk and limb ischemia on the one hand and elevated glycosylated HbA1c on the other has been confirmed in many studies, which showed that an $\mathrm{HbA1c}$ level of $6 \%$ or more is a good clinical predictor of developing cardiovascular disease and even death and that HbA1c levels of $7.5 \%$ or more in people with diabetes are associated with more than five times higher risk of being hospitalized with the peripheral arterial disease compared with patients with controlled diabetes $[11,13]$. Other observations include a link between high HbA1c levels and infection rates (e.g., skin, cellulitis, candidiasis, bone, and joints), which tend to rise with elevated HbA1c and are the main cause of admission of people with diabetes $[9,14,15]$.

Our study is a single-center study, so its findings cannot be generalized. Also, its retrospective design makes it prone to bias and missing data. In addition, we were unable to control for the accuracy of the HbA1c tests as they were done in different labs due to a shortage of resources. Moreover, we could not exclude false high HbA1c values due to anemia, as many patients included in this study had iron deficiency anemia, and also due to stress hyperglycemia, which is encountered frequently in inpatients. Other factors that can affect the sensitivity of the HbA1c test are abnormal hemoglobin kinetics and possible blood transfusion, the latter of which cannot be excluded.

\section{Conclusion}

HbA1c correlated significantly with hospitalization in type- 1 but not in type- 2 diabetes. HbA1c measurement policy for hospitalized patients with diabetes mellitus is crucial as it is a predictor of readmission rate, especially in people with type 1 diabetes.

\section{Acknowledgment}

This paper and the research behind it would not have been possible without the exceptional support of my supervisor, Faiza Hander. Her enthusiasm, knowledge, and exacting attention to detail have been an inspiration and kept my work on track Laila Dabaj my colleagues, have also looked over my transcriptions and answered with unfailing patience numerous questions about the language, I am also grateful for the insightful comments offered by the anonymous peer reviewers. The generosity and expertise of one and all have improved this study in innumerable ways and saved me from many errors; those that inevitably remain are entirely my own responsibility.

I fully declare any financial or another potential conflict of interest.

\section{References}

1. Kornum JB, Thomsen RW, Riis A, Lervang HH, Schønheyder HC, et al. Diabetes, Glycemic Control, and Risk of Hospitalization With Pneumonia. Diabetes Care. 2008; 31: 1541-1545.

PubMed: https://pubmed.ncbi.nlm.nih.gov/18487479/

2. Mazurek JA, Hailpern SM, Goring T, Nordin C. Prevalence of hemoglobin A1c greater than $6.5 \%$ and $7.0 \%$ among hospitalized patients without known diagnosis of diabetes at an Urban Inner City Hospital. J Clin Endocrinol Metab. 2010; 95: 1344-1348. PubMed: https://pubmed.ncbi.nlm.nih.gov/20080838/

3. Wexler DJ, Nathan DM, Grant RW, Regan S, Van Leuvan AL, et al. Prevalence of elevated hemoglobin A1c among patients admitted to the hospital without a diagnosis of diabetes. J Clin Endocrinol Metab. 2008; 93: 4238-4244.

PubMed: https://pubmed.ncbi.nlm.nih.gov/18697862/

4. Greci LS, Kailasam M, Malkani S, Katz DL, Hulinsky I, et al. Utility of $\mathrm{HbA} 1 \mathrm{c}$ levels for diabetes case finding in hospitalized patients with hyperglycemia. Diabetes care. 2003; 26: 1064-1068.

PubMed: https://pubmed.ncbi.nlm.nih.gov/12663574/

5. Hemmingsen B, Lund SS, Gluud C, Vaag A, Almdal T, et al. Intensive glycaemic control for patients with type 2 diabetes: Systematic review with meta-analysis and trial sequential analysis of randomised clinical trials. BMJ. 2011; 343: 1136.

PubMed: https://pubmed.ncbi.nlm.nih.gov/22115901/

6. Patel A, MacMahon S, Chalmers J. Action in Diabetes and Vascular Disease: Preterax and Diamicron Modified Release and Controlled Evaluation (ADVANCE) Collaborative Group. Intensive blood glucose control and vascular outcomes in patients with type 2 diabetes. $\mathrm{N}$ Engl J Med. 2008; 358: 2560-2572.

7. NG A, Sandra JG, Shreekant P. Glycemic Control and Risk of Cardiovascular Disease Hospitalization and All-Cause Mortality. J Am Coll Cardiol. 2013; 62: 121-127.

PubMed: https://pubmed.ncbi.nlm.nih.gov/23665365/

8. Shi L, Ye X, Lu M, Wu EQ, Sharma H, et al. Clinical and economic 
benefits associated with the achievement of both hba1c and LDL cholesterol goals in veterans with type 2 diabetes. Diabetes Care. 2013; 36: 3297-3304.

PubMed: https://pubmed.ncbi.nlm.nih.gov/23801723/

9. Critchley JA, Carey IM, Harris T, DeWilde S, Hosking FJ, et al Glycemic control and risk of infections among people with type 1 or type 2 diabetes in a large primary care cohort study. Diabetes Care. 2018;41: 2127-2135.

PubMed: https://pubmed.ncbi.nIm.nih.gov/30104296/

10. Currie CJ, Peters JR, Tynan A, Evans M, Heine RJ, et al. Survival as a function of $\mathrm{HbA} 1 \mathrm{C}$ in people with type 2 diabetes: a retrospective cohort study. Lancet. 2010; 375: 481-489.

PubMed: https://pubmed.ncbi.nIm.nih.gov/20110121/

11. Menzin J, Korn JR, Cohen J, Lobo F, Zhang B, et al. Relationship between glycemic control and diabetes-related hospital costs in patients with type 2 diabetes mellitus. J Manag Care Pharm. 2010; 16: 264-2675. PubMed: https://pubmed.ncbi.nlm.nih.gov/20433217/
12. Yu D, Simmons D. Relationship between $\mathrm{HbA} 1 \mathrm{c}$ and risk of all-cause hospital admissions among people with Type 2 diabetes. Diabet Med. 2013; 30: 1407-1411.

PubMed: https://pubmed.ncbi.nlm.nih.gov/23692400/

13. Selvin E, Wattanakit K, Steffes MW, Coresh J, Sharrett AR. HbA1c and peripheral arterial disease in diabetes: The atherosclerosis risk in communities study. Diabetes Care. 2006; 29: 877-882. PubMed: https://pubmed.ncbi.nlm.nih.gov/16567831/

14. Haifa elhadi A, Faiza $H$. Reasons for admission of individual with diabetes to the Tripoli Medical Center in 2015. Diabetes Metab Syndr Clin Res Rev. 2019; 13: 2571-2578.

PubMed: https://pubmed.ncbi.nlm.nih.gov/31405678/

15. Umpierrez GE, Hellman R, Korytkowski MT, Kosiborod M, Maynard GA, et al. Management of hyperglycemia in hospitalized patients in noncritical care setting: An endocrine society clinical practice guideline. $J$ Clin Endocrinol Metab. 2012; 97: 16-38.

PubMed: https://pubmed.ncbi.nlm.nih.gov/22223765/ 